including primary biliary cirrhosis. IL-17 related cytokines have multiple effects on parenchymal cells in different tissues and may be involved in both effector responses and repair and regeneration. We recently reported increased numbers of liver infiltrating IL-17 secreting CD4 and CD8 T cells in chronic inflammatory liver disease. These cells also secrete IL-21 and IL-22 cytokines reported to promote epithelial repair. In the present study we report the pattern of IL-17, IL-21 and IL-22 receptor expression on hepatic parenchymal cells and demonstrate cell-specific effects of $T_{h 17}$ cytokines on these target cells.

Aim To assess the functional impact of Th17/Tc17 associated cytokines on hepatic parenchymal cells.

Method Primary human cholangiocytes, hepatocytes and sinusoidal endothelial cells were assessed for IL-17, IL-21 and IL-22 receptor expression. The effects of stimulation with recombinant IL-17, IL-21, IL-22, TNF-a or IFN-g alone or in combination were compared on apoptosis, proliferation and cytokine secretion using flow cytometry with annexin or 7-AAD staining and in situ Ki67 staining and measurement of IL-1b, IL-6, IL-23 and TGF-b1 secretion by ELISA.

Results All the parenchymal cell types expressed IL-21R and IL-22R. Th17 and Tc17 cytokines did not cause apoptosis but alone and in combination led to parenchymal cell proliferation. Cholangiocytes and hepatocytes responded best to IL-17, whereas sinusoidal endothelial cells were responsive to IL-22. Cholangiocytes responded to Th17/Tc17 cytokines by secreting high levels of IL-1b, IL-6, IL-23 and TGF-b1 all cytokines that support the survival of Th17 and Tc17 cells.

Conclusion Liver parenchymal cells express IL-17, IL-21 and IL-22 receptors and proliferate in response to Th17/Tc17 cytokines. Cholangiocytes also respond to such cytokines by secreting Th17 polarising cytokines. Thus IL-17 related cytokines secreted by infiltrating lymphocytes may activate the epitheliome to generate a local environment characterised by cholangiocyte proliferation and Th17 cell survival. This response may contribute to the bile duct proliferation and persistent chronic inflammation that characterised many liver diseases.

\section{P99 FXR STIMULATION WITH INT-747 IN A RAT BILIARY DRAINAGE MODEL PROTECTS FROM HEPATOCELLULAR INJURY AFTER LOSS OF ENTEROHEPATIC CIRCULATION}

doi:10.1136/gutjnl-2011-300857a.99

\begin{abstract}
${ }^{1}{ }^{2} \mathrm{R}$ G J Visschers, ${ }^{1}{ }^{2} \mathrm{~K}$ V K Koelfat, ${ }^{1}{ }^{2} \mathrm{C} \mathrm{M}$ Hodin, ${ }^{2}{ }^{3} \mathrm{R}$ Shiri-Sverdlov, ${ }^{1}$ ${ }^{2}$ W A Buurman, ${ }^{4} \mathrm{R}$ Mookerjee, ${ }^{12} \mathrm{~K}$ Lenaerts, ${ }^{1}{ }^{2}{ }^{5} \mathrm{~S}$ W M 0 Damink. ${ }^{1}$ Department of Surgery, Maastricht University Medical Centre +, Maastricht University, Maastricht, the Netherlands; ${ }^{2}$ Nutrition and Toxicology Research Institute Maastricht, Maastricht University, the Netherlands; ${ }^{3}$ Department of Molecular Genetics, Maastricht University Medical Centre+, the Netherlands; ${ }^{4}$ UCL Hepatology, Royal Free Hospital, University College London, London, UK; ${ }^{5}$ Department of HPB Surgery and Liver Transplantation, Royal Free Hospital, University College London, London, UK
\end{abstract}

Introduction Enterocutaneous fistula are often associated with development of intestinal failure associated liver disease (IFALD), ultimately leading to liver damage. We hypothesise that this is caused by reduced farnosoid X receptor (FXR) stimulation, due to interruption of the enterohepatic circulation and consequent impact on bile acid synthesis.

Aim We aimed at investigating the effect of specific stimulation of the farnosoid X receptor (FXR) with INT-747, a synthetic agonist, in a rat biliary diversion model.

Methods Four groups of rats $(n=6-8)$ were studied; two groups underwent bile duct cannulation and externalisation to achieve continuous biliary drainage, daily receiving either vehicle or INT-747 (Intercept Pharm LtD); two groups underwent laparotomy without cannulation also receiving either vehicle or INT-747. Loss of bile was recorded daily. After 7 days, plasma, serum and liver and intestinal tissue were collected. Alanine aminotransferase (ALT), aspartate aminotransferase (AST), alkaline phosphatase (AP), $\gamma$ glutamyl transpeptidase (GGT) and total bilirubin were assessed, together with histological scoring of necrosis, inflammation, bile duct proliferation, fibrosis and steatosis. Expression of genes involved in the FXR pathway, and lipid and cholesterol metabolism were quantified by qPCR.

Results Loss of bile was significantly reduced in the INT-747 group compared to the vehicle group. Serum levels of ASAT, ALAT, AP, GGT, bilirubin (total and direct) were all significantly increased in the bile drainage group when compared to controls $(p<0.05)$, suggesting hepatocellular damage. Interestingly, all parameters were significantly decreased in the bile drainage group receiving INT-747 $(p<0.05)$. Histological analyses showed normal liver histology in both sham groups. In contrast, large necrotic areas were observed in the biliary diversion group receiving vehicle with a high number of infiltrating inflammatory cells, which decreased significantly in the biliary diversion group receiving INT-747 $(p<0.05)$. Biliary diversion induced hepatic fibrosis and bile duct proliferation, which were both attenuated by INT-747 supplementation $(p<0.05)$. Although genes involved in the FXR pathway (FXR, SHP, CYP7A1 and CYP8B1) were influenced by bile drainage, no significant change was observed when rats received INT-747.

Conclusion The present data demonstrate that stimulation of FXR with INT-747 can attenuate hepatocellular damage in an experimental model of IFALD. The results suggest a role of FXR in the development of hepatocellular damage, hepatic fibrosis and necrosis during biliary diversion. FXR stimulation has the potential to be a novel therapy for patients with IFALD.

\section{P100 LIVER TRANSPLANTATION (LT) RESULTS IN REDUCED RECIPIENT NATURAL KILLER (NK) CELL ACTIVATION WITH ASSOCIATED DOWN REGULATION OF ACTIVATING RECEPTORS NKP3O AND NKP46 BUT NOT NKG2D}

doi:10.1136/gutjnl-2011-300857a.100

${ }^{1} \mathrm{~K}$ M Jamil, ${ }^{1} \mathrm{~K}$ S Cheent, ${ }^{2} \mathrm{~K}$ Agarwal, ${ }^{3} \mathrm{G}$ J Alexander, ${ }^{2} \mathrm{M}$ A Heneghan, ${ }^{1} \mathrm{~S}$ I Khakoo. ${ }^{1}$ Imperial College London; ${ }^{2}$ King's College Hospital, London; ${ }^{3}$ Addenbrookes Hospital, Cambridge

Introduction In solid organ transplantation, the effect of the allograft on recipient NK cell function is poorly understood. NK cells recognise self through inhibitory receptors for HLA class I, so that they

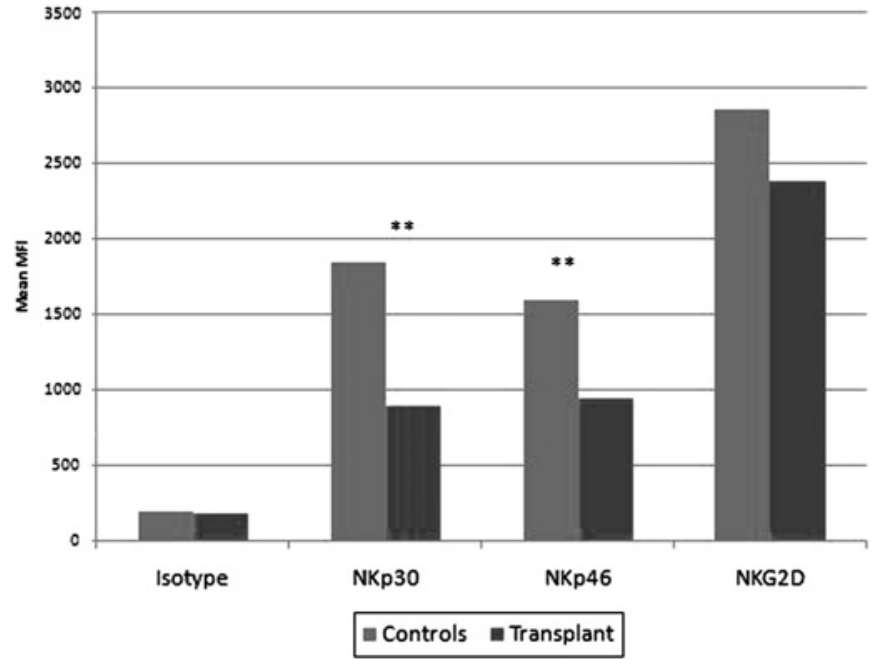

Abstract P100 Figure 1 Expression of activating receptors on NK cells. 


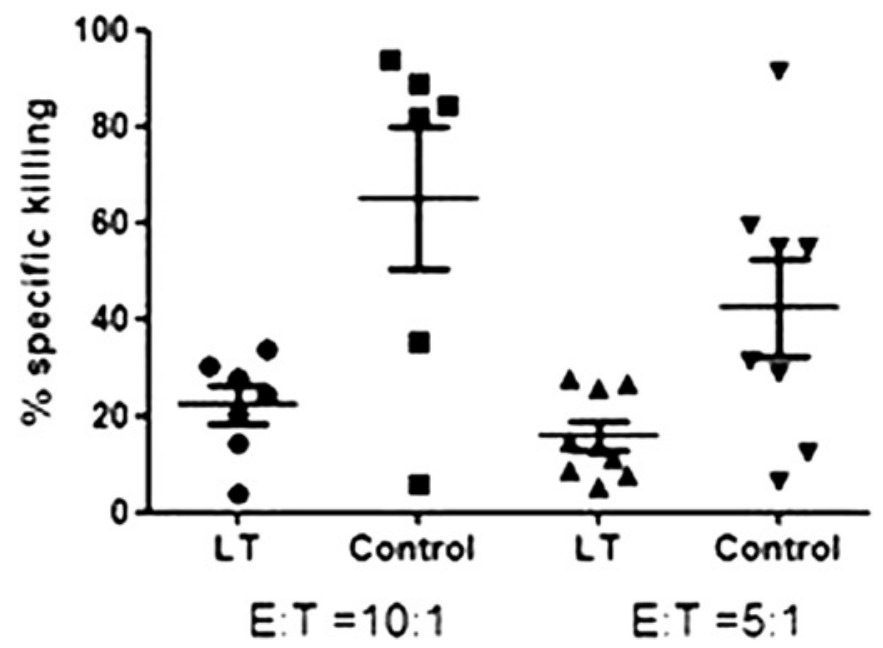

Abstract P100 Figure 2 NK cell Killing of K562 target cells.

lyse target cells that do not express self HLA class I molecules (the "missing-self" model). As liver transplants are not matched for HLA, significant NK cell alloreactivity is expected, but is not seen in practise.

Aim This aim of this study is to investigate the effect of LT on recipient NK cell reactivity.

Method Whole blood was collected from 16 liver transplant patients and 10 controls. The transplant patients were all on calcineurin inhibitor-based immunosuppression. NK cells from the peripheral blood were analysed for expression of the cell surface inhibitory receptors CD158a/b (killer cell immunoglobulin-like receptors (KIR) specific for HLA-C), and the activating receptors NKp30, NKp46 and NKG2D. Following overnight incubation with IL-15 NK cell function was assessed using a flow cytometry-based killing assay.

Results There was significantly reduced expression of NKp30 and NKp46 in post LT patients compared with controls $(p<0.001$ for both, Student $t$ test), but no reduction in NKG2D expression (Abstract P100 figure 1). There was no difference in KIR expression or segregation of activating receptor expression with expression of specific KIR. However, consistent with the phenotyping results there was a significant reduction of NK cell killing of target cells in post LT patients compared with controls as shown in Abstract P100 figure 2 ( $p=0.011$, Student $t$ test $)$.

Conclusion Following liver transplantation there is down-regulation of activating NK cell receptors and suppression of NK cell activity. We propose that this suppression helps to maintain tolerance of the HLA-mismatched liver allograft.

\section{P101 REGULATORY T CELLS EXHIBIT REDUCED PHENOTYPIC STABILITY UPON PRO-INFLAMMATORY CHALLENGE IN AUTOIMMUNE HEPATITIS}

doi:10.1136/gutjnl-2011-300857a.101

${ }^{1} \mathrm{C}$ R Grant, ${ }^{1} \mathrm{~B}$ Holder, ${ }^{1} \mathrm{Y}$ Ma, ' $\mathrm{G}$ Mieli-Vergani, ${ }^{1} \mathrm{D}$ Vergani, ${ }^{1} \mathrm{M}$ S Longhi. ${ }^{1}$ King's College London School of Medicine at King's College Hospital

Introduction In autoimmune hepatitis (AIH) $\mathrm{CD} 4{ }^{+} \mathrm{CD} 25^{\text {high }}$ regulatory T-cells (Tregs) are numerically defective and fail to suppress T-cell-mediated immune responses. Expression by Tregs of the ectoenzyme CD39 contributes to their ability to suppress by initiating an ATP hydrolysis cascade which leads to the production of adenosine, a molecule with inhibitory properties. Recently expression of CD39 has been associated with T-reg phenotypic stability under inflammatory conditions.

Aim To investigate the frequency and phenotypic stability of $\mathrm{CD}^{+} 9^{+}$Tregs in AIH.

Method 24 AIH patients (23 ANA/SMA ${ }^{+}, 1 \mathrm{LKM}^{-1}{ }^{+}$; 12 females, median age: 15 years) and 24 healthy subjects (HS; 16 females, median age: 35 years) were studied. The phenotype of circulating Tregs was assessed by flow cytometry using monoclonal antibodies to CD4, CD25, CD127, CD39 and CD73, an ectonucleotidase that in mice is expressed by Tregs and works in tandem with CD39. The frequency of IFN $\gamma$, IL10 and IL17-producing cells within Tregs was determined by intracellular staining. Analysis was performed at baseline and after exposure to anti-CD3/CD28 T-cell expander or to the pro-inflammatory cytokines IL1 $\beta$ and IL6 (IL1 $\beta+$ IL6).

Results At baseline, CD $39^{+}$Tregs were less numerous in $\mathrm{AIH}$ $(8.78 \pm 0.77)$ than HS $(11.93 \pm 1.04, \mathrm{P}=0.019)$ and displayed a trend towards higher CD127 expression ( $7.41 \pm 3.25$ vs $2.75 \pm 1.33$, $\mathrm{p}=0.13$ ) and reduced FOXP3 mean fluorescence intensity $(1230 \pm 260$ vs $997 \pm 232, p=0.09)$. CD73 expression on $\mathrm{CD}^{2} 9^{+}$Tregs did not differ between the two groups. Exposure to T-cell expander increased the frequency of IFN $\gamma^{+} \mathrm{CD} 39^{+}$Tregs in $\mathrm{AIH}$ (from $7.37 \pm 2.15$ to $24.3 \pm 13.37, \mathrm{p}=0.043)$ but not in HS $(12.4 \pm 2.9$ to $6.6 \pm 2.4, \mathrm{p}=\mathrm{NS})$. Although the frequency of IFN $\gamma^{+} \mathrm{CD} 39^{+}$Tregs augmented after treatment with IL1 $\beta+$ IL6 in both AIH (from $7.37 \pm 2.15$ to $72.9 \pm 9.42$, $\mathrm{p}<0.001)$ and HS $(12.39 \pm 2.94$ to $56.51 \pm 16.12, \mathrm{p}<0.001)$, the increase was higher in the former than in the latter (10-fold vs fivefold). IL1 $\beta$ + IL6 increased the frequency of $\mathrm{CD} 127^{+} \mathrm{CD} 39^{+}$Tregs in AIH (from $4.95 \pm 2.23$ to $19.6 \pm 11.43, p=0.05)$ but not in HS $(2.7 \pm 1.3$ to $1.3 \pm 0.8$ $\mathrm{p}=\mathrm{NS}$ ). No change in the frequency of IL $10^{+}$and $\mathrm{IL} 17^{+} \mathrm{CD} 39^{+}$Tregs was noted upon T-cell expander or IL1 $\beta+$ IL6 stimulation in AIH and $\mathrm{HS}$

Conclusion Compared to HS, Tregs from AIH patients display lower CD39 expression and are more prone to become activated upon exposure to pro-inflammatory stimuli, a finding which indicates reduced phenotype stability. A decrease in CD39 expression and in phenotypic stability may contribute to impaired Treg suppressive function in $\mathrm{AIH}$

\section{P102 CLEVER-1 MEDIATES THE TRANSMIGRATION OF B CELLS ACROSS HUMAN HEPATIC SINUSOIDAL ENDOTHELIUM}

doi:10.1136/gutjnl-2011-300857a.102

${ }^{1} \mathrm{~S}$ Shetty, ${ }^{1} \mathrm{C}$ Weston, ${ }^{1} \mathrm{Y}$ Oo, ${ }^{1} \mathrm{Z}$ Stamataki, ${ }^{2} \mathrm{M}$ Salmi, ${ }^{2} \mathrm{~S}$ Jalkanen, ${ }^{1} \mathrm{P}$ Lalor, ${ }^{1} \mathrm{D}$ Adams. ${ }^{1}$ University of Birmingham; ${ }^{2}$ University of Turku

Introduction Lymphocytes are recruited via the unique hepatic sinusoidal channels during chronic inflammatory liver diseases. This low shear vascular bed is lined by hepatic sinusoidal endothelium (HSEC) which lacks certain conventional adhesion molecules leading us to look for novel receptors involved in lymphocyte recruitment. HSEC express several scavenger receptors including CLEVER-1 which we have recently shown mediates regulatory $\mathrm{T}$ cell recruitment to HSEC. B cells have been implicated in the pathogenesis of liver disease and driving liver fibrosis.

Aim B cells must be recruited from the peripheral circulation into liver tissue but the molecular mechanisms that mediate this process are not known. Our aim was to study if CLEVER-1 plays a role in this process.

Method We used isolated HSEC in flow adhesion assays to study the functional role of CLEVER-1 in lymphocyte subset recruitment. Immunofluorescent staining and confocal microscopy were used to characterise the transmigration of lymphocytes across HSEC under conditions of flow. Time lapse video recordings and Image J software was used to compare $\mathrm{T}$ cell and $\mathrm{B}$ cell recruitment via HSEC monolayers under conditions of flow. 